\title{
Growth performance, carcass and meat quality of lambs supplemented different vegetable oils
}

\author{
Renata Miltko', Małgorzata Paulina Majewska', Grzegorz Bełżecki', \\ Katarzyna Kula', and Barbara Kowalik ${ }^{1, *}$
}

\begin{abstract}
* Corresponding Author: Barbara Kowalik Tel: +48-227653335, Fax: +48-227653302, E-mail: b.kowalik@ifzz.pl
\end{abstract}

${ }^{1}$ The Kielanowski Institute of Animal Physiology and Nutrition, Polish Academy of Sciences, Jabłonna 05-110, Poland

ORCID

Renata Miltko

https://orcid.org/0000-0001-6835-4807 Małgorzata Paulina Majewska

https://orcid.org/0000-0002-6078-9678 Grzegorz Bełżecki

https://orcid.org/0000-0003-2237-1454 Katarzyna Kula

https://orcid.org/0000-0003-4027-7090

Barbara Kowalik

https://orcid.org/0000-0003-3884-1967

Submitted Jun 28, 2018; Revised Sept 9, 2018; Accepted Nov 5, 2018
Objective: The purpose of this study was to evaluate the effect of rapeseed and linseed oil supplementations on performance and meat quality of lambs.

Methods: The experiment was conducted on 18 growing (100-day-old) lambs of $19.7 \pm 1.9 \mathrm{~kg}$ live weight, assigned to 3 groups of 6 animals each. Control lambs were fed meadow hay and concentrate alone. Experimental animals additionally received rapeseed or linseed oils at a dose of $50 \mathrm{~g} / \mathrm{d}$. The lambs were slaughtered at an average body weight of $35.7 \pm 0.5 \mathrm{~kg}$.

Results: The dressing percentage was higher in lambs fed rapeseed oil. Total saturated fatty acids (SFA) and C15:0, C16:0, C17:0, C21:0, C24:0 were lower in longissimus dorsi muscle (MLD) in lambs fed linseed oil. Supplementation of diet with linseed oil decreased concentrations of total monounsaturated fatty acids and C16:1, C17:1, C18:1 cis-9 in MLD. The concentrations of $n-3$ polyunsaturated fatty acids (PUFA) and C18:3n-3, C20:5 n-3 in MLD were higher in lambs fed linseed oil than in other groups. Oils supplementation to diets resulted in increased concentration of C22:6n-3 in MLD. The inclusion of linseed oil into the diet increased the contents of total PUFA, $n-3$ PUFA and C18:3 n-3, C20:5 n-3, C22:6n-3 in semitendinosus muscle in comparison to control. A tendency towards a lower n:6/n:3 ratio in MLD was observed when lambs were supplemented linseed oil.

Conclusion: The supplementation of linseed oil to diets seems to reduce the concentration of SFA and increase the concentration of $n-3$ PUFA. The $n-6 / n-3$ ratio is an important nutritional factor, and its value has been favorably decreased below 2 , thereby achieving an important target related to human health. Due to these changes carcass fatty acid profile was improved, and so enhanced lamb meat healthy properties.

Keywords: Lamb Meat; Rapeseed Oil; Linseed Oil; Fatty Acids

\section{INTRODUCTION}

Ruminant meat is an important dietary component of human diets. Lamb and beef meat have been associated with an increased risk of cardiovascular diseases due to their high content of saturated fatty acid (SFA) [1]. The formation of large SFA is a result of the biohydrogenation process in the rumen during which bacteria convert unsaturated fatty acid (USFA) to SFA. So, the fatty acids (FA) occurring in the rumen are highly saturated and take part in the absorption as well as deposition of the fat in muscles. Nevertheless, such meat may be also a good dietary source of some nutrients with health benefits, including certain FA, particularly polyunsaturated fatty acids ( $\mathrm{PUFA} \geq \mathrm{C} 20$ ) as well as conjugated linoleic acid (CLA) isomers. In addition, in a review by Chikwanha et al [2] it was shown that detrimental effects of rations rich in monounsaturated fatty acids (MUFA) are not known. In another study it was reported that oleic acid C18:1 cis-9, the primary $c$-MUFA found in lamb meat, was associated with beneficial effects related to cancer prevention as well as inflammatory and 
autoimmune diseases [3].

The decrease in SFA and increase of health-beneficial FA (PUFA and CLA) contents have been an important objective of ruminant meat studies. According to WHO/FAO [4], the ratio of PUFA and SFA is a significant indicator for nutritional evaluation of fat, with a recommendation of about 0.40 . Beneficial effects of PUFA, such as eicosapentaenoic (EPA, C20:5 $n$-3) and docosahexaenoic (DHA, C22:6n-3) acids, include anti-inflammatory, anti-atherogenic and anti-thrombotic actions [5]. It is essential to increase the consumption of EPA and DHA in human diets, because the synthesis of these FAs from dietary a-linolenic acid (ALA, C18:3 n-3) is very restricted.

In addition, lamb meat is among the richest natural sources of CLA. Many studies have shown that CLA demonstrate anticarcinogenic, anti-diabetogenic and anti-atherogenic effects as well as other health-promoting properties [6]. Of twentyeight known CLA isomers, cis9 trans-11 (rumenic acid) is the most common isomer exhibiting the highest biological activity. This isomer is formed during ruminal biohydrogenation of linoleic acid (LA, C18:2 n-6) to stearic acid (C18:0) and by endogenous conversion of vaccenic acid (VA, C18:1 trans-11) by $\Delta 9$ desaturase in tissues [7].

Some nutritional aspects, for example FA profile of the ration, lipid supplementation source, forage type as well as the forage/concentrate ratio in the diet are main driving factors of ruminant meat [2]. In ruminants some sources of FA such as ground oilseeds [8], whole oilseeds [9,10], extruded oilseeds [11] have already been studied. It was shown that the FA profile and concentration of CLA in meat may be affected by the form of the provided lipid. However, experiments evaluating kinds of oils are scarce $[12,13]$ and the exact influence of particular form of fat on FA profile of lamb and beef meat is limited.

It was hypothesised that diet supplementation with rapeseed oil rich in oleic acid, or linseed oil, a good source of linolenic acid, can reduce the concentration of SFA and increase $n-3$ PUFA.

So, the objective of this study was to compare the effect of rapeseed and linseed oils on lamb growth, carcass traits and FA composition of longissimus dorsi and semitendinosus muscles (MS) with implications to human health.

\section{MATERIALS AND METHODS}

All procedures were approved by the Local Ethics Committee for Animal Experimentation in Warsaw (No 51/2009).

\section{Animals and feed}

The study was conducted on 18 growing (100-day-old) male Corridale lambs of $19.7 \pm 1.9 \mathrm{~kg}$ live weight. The animals were assigned to 3 groups of 6 lambs each (according to initial body weight) in a randomized complete block design. The animals
Table 1. Composition of diets

\begin{tabular}{lcc}
\hline \multirow{2}{*}{ Items } & \multicolumn{2}{c}{ Diet } \\
\cline { 2 - 3 } & Control & Oil $^{1)}$ \\
\hline Ingredients (g/kg DM) & \\
$\quad$ Meadow hay & 369 & 350 \\
Crushed barley & 452 & 431 \\
Soybean oilmeal & 159 & 152 \\
Oil & - & 48 \\
Vitamin-mineral premix ${ }^{2)}$ & 20 & 19 \\
Chemical composition (g/kg DM) & & \\
DM (g/kg) & 874 & 899 \\
Crude protein & 175 & 164 \\
Crude fat & 22.9 & 69 \\
Starch & 342 & 324 \\
Crude ash & 45.4 & 43.1 \\
NDF & 442 & 421 \\
ADF & 191 & 182 \\
UFV (kg) & 1.0 & 1.1 \\
\hline
\end{tabular}

DM, dry matter; NDF, neutral detergent fibre; ADF, acid detergent fibre; UFV, feed unit of maintenance and meat production.

1) Rapeseed or linseed oils.

2) Contained per kg: vit $\mathrm{A}$ 300,000 IU; vit $\mathrm{D}_{3} 30,000 \mathrm{IU}$; vit $\mathrm{E} 1.5 \mathrm{~g}$; Mn 3 g; Zn 2.5 g; Co 0.015 g; Se 0.003 g; Ca 240 g; Na 60 g; P 120 g; Mg 65 g.

were housed in individual pens on wheat straw with separate facilities for feeding. Control animals were fed a basal diet containing meadow hay, concentrate - crushed barley and soybean oilmeal and vitamin-mineral mixture (Polfamix - OK, Trouw Nutrition, Poland); experimental lambs were fed a basal ration and 5\% rapeseed or linseed oils. Oils were stored out of direct sunlight at $4^{\circ} \mathrm{C}$ and supplements were mixed into the concentrate. Dietary compositions are given in Table 1 and FA profiles of rapeseed and linseed oils are presented in Table 2. Daily rations were offered in two equal portions at 8:00 and 16:00. The lambs had free access to water and blocks of salt containing microelements. Since animals were provided with a limited amount of feed, no orts were observed.

The animals were weighed every two weeks during fattening in order to adjust diets to body weight. The lambs were weighed just before morning feeding. Feed intake, utilization as well as daily live weight gains were recorded. Daily feed intake was compared with the requirement for $250 \mathrm{~g}$ gain and adjusted to the average daily gain (ADG) obtained for each

Table 2. Contents of some fatty acids in rapeseed and linseed oils (g FAME/100 g oil)

\begin{tabular}{lcc}
\hline Fatty acid & Rapeseed oil & Linseed oil \\
\hline C16:0 palmitic & 1.9 & 1.9 \\
C18:0 stearic & 2.3 & 1.6 \\
C18: 1 n-9 oleic & 63.7 & 28.5 \\
C18:2 $n$-6 linoleic & 23.0 & 17.5 \\
C18:3 $n$-3 linolenic & 8.8 & 50.1 \\
\hline
\end{tabular}

FAME, fatty acid methyl esters. 
$\operatorname{diet}[14]$.

\section{Slaughter and carcass measurements}

The lambs were slaughtered after about 55 days at an average body weight of $35.7 \pm 0.5 \mathrm{~kg}$, in accordance with standard practises [15]. Before slaughter, animals were starved for $12 \mathrm{~h}$, but had free access to drinking water. Afterwards, non-carcass components, gastrointestinal tracts and viscera were removed. Selected internal organs (liver, heart, and kidneys) were weighted. Carcasses were kept at $4^{\circ} \mathrm{C}$ for $24 \mathrm{~h}$ and a simple dissection of their right halves was carried out. Thereafter, carcasses were split into commercial cuts, i.e. loin, shoulder and leg; selected cuts were weighted. The samples of longissimus dorsi muscle (MLD) from the 10th to 13th rib and MS were taken to analyse the chemical composition and FA profile. Muscle samples were minced, packed into polyethylene bags and stored at $-30^{\circ} \mathrm{C}$ until analysis.

\section{Chemical analysis}

The chemical composition of feed and muscle samples was determined according to standard methods [16]. The samples were analysed for dry matter (DM) (AOAC official method 934.01), crude protein (CP, total nitrogen $\times 6.25$, AOAC official method 954.01), crude fat (AOAC official method 960.39), ash (AOAC official method 930.05). Starch concentration in feeds was determined according to the official method of AOAC 920.40; natural detergent fibre (NDF) and acid detergent fibre $(\mathrm{ADF})$ were analysed according to van Soest et al [17].

Lipids from oils and muscles were extracted with chloroformmethanol (2:1), according to Folch et al [18]. After filtration through a filter paper, $800 \mu \mathrm{L}$ of filtrate was transferred into glass vials. Thereafter, vials were evaporated to dryness under nitrogen stream in a heating block at $50^{\circ} \mathrm{C}$. The samples were saponified with $1.5 \mathrm{~mL}$ of $0.5 \mathrm{M} \mathrm{KOH}$ in methanol in a heating block at $75^{\circ} \mathrm{C}$. Next, the samples were esterified with 3.0 $\mathrm{mL}$ of $4 \%$ solution of $\mathrm{SOCl}_{2}$ in methanol. Methyl esters were extracted with $1.0 \mathrm{~mL}$ of $\mathrm{n}$-heptane and salted out with $\mathrm{NaCl}$ to separate the organic layer. Subsequently, $300 \mu \mathrm{L}$ methyl esters were transferred into glass vials and $600 \mu \mathrm{L}$ of $\mathrm{n}$-heptane was added. The vials were kept at $-30^{\circ} \mathrm{C}$ until fatty acid methyl esters (FAME) analyses. The FAME were separated and quantified using a gas chromatograph (Shimadzu GC-2010, Kyoto, Japan) equipped with a flame-ionisation detector (FID), injection port and a BPX70 fused silica capillary column (120 m $\times 0.25 \mathrm{~mm}$ i.d. $\times 0.25 \mu \mathrm{m}$ film thickness [SHIM-POL, Izabelin, Poland]). Helium was used as the carrier gas, operated at a constant pressure of $223.4 \mathrm{kPa}$ and flow rate of $1 \mathrm{~mL} / \mathrm{min}$. Injector and FID temperatures were maintained at $200^{\circ} \mathrm{C}$ and $240^{\circ} \mathrm{C}$, respectively. FAME profile was analysed using the temperature gradient programme in a $1-\mu \mathrm{L}$ sample at a split ratio of 10:1, as described by Czauderna et al [19]. The analysis started at $70^{\circ} \mathrm{C}$ for $4 \mathrm{~min}$ increasing at a rate of $12^{\circ} \mathrm{C} / \mathrm{min}$ to $150^{\circ} \mathrm{C}$, which was maintained for $6 \mathrm{~min}$; then increased at $8^{\circ} \mathrm{C} /$ min to $168^{\circ} \mathrm{C}$, maintained for $27 \mathrm{~min}$ and increased by $0.75^{\circ} \mathrm{C} / \mathrm{min}$ to $190^{\circ} \mathrm{C}$ and maintained for $10 \mathrm{~min}$; then increased again at a rate of $1.8^{\circ} \mathrm{C}$ to $210^{\circ} \mathrm{C}$, maintained for 15 $\mathrm{min}$; elevated at a rate of $6^{\circ} \mathrm{C} / \mathrm{min}$ to $234^{\circ} \mathrm{C}$, which was kept for $4 \mathrm{~min}$ and finally increased at a rate of $6^{\circ} \mathrm{C} / \mathrm{min}$ to 236 and maintained for $20 \mathrm{~min}$. FAME peaks were identified by comparison with Supelco 37 Component FAME Mix (Supelco Inc., Bellefonte, PA, USA) and CLA standard mix (Sigma-Aldrich, Inc., St. Louis MO, USA).

The $\Delta^{9}$-desaturase index was calculated as $(\mathrm{C} 14: 1$ cis-9+C16:1 cis-9+C18:1 cis-9+C18:2 cis-9, trans-11)/(C10:0+C14:0+C14:1 cis-9+C16:0+C16:1 cis-9+C18:0+C18:1 cis-9+C18:1 trans-11+ C18:2 cis-9, trans-11), according to Brogna et al [20].

\section{Statistical analysis}

Data were expressed as means and pooled standard error of the mean. The normality of the data and homogeneity of variance were tested using the Shapiro-Wilk and Brown-Forsythe tests, respectively. Data were analyzed using one-way analysis of variance in the general linear model of Statistica (Statistica 10.0 software, StatSoft, Kraków, Poland) with treatment (diet) as a fixed effect.

The effects were considered to be significant at $\mathrm{p} \leq 0.05$ or $p<0.01$ and $p$ values $<0.10$ were considered trends in the data. The individual means were compared by the Tukey HSD posthoc test (Statistica 10.0 software, StatSoft, Poland).

\section{RESULTS}

\section{Animal performance}

Nutrient composition of the diets is given in Table 1. As expected, crude fat content and feed unit of maintenance and meat production were higher for oil-supplemented than control ration. Furthermore, the contents of $\mathrm{CP}$, crude ash and starch as well as NDF and ADF (as g/kg DM) were lower for oil-supplemented than control diet. Daily DM intake was higher when oils were supplemented in comparison to the control ration (1.05 vs $1.00 \mathrm{~kg} \mathrm{DM} / \mathrm{d})$.

Lambs in different oil supplementation treatments were similar in terms of ADG ( $p=0.816)$ and feed conversion ratio $(\mathrm{p}=0.982)$ (Table 3$)$. Significantly higher $(\mathrm{p}=0.039)$ dressing percentage (DP) and a tendency towards increased hot carcass and right half carcass weights $(p=0.100$ and $p=0.060$, respectively) were recorded in lambs fed the rapeseed oil diet. The weights of carcass components, i.e. loin ( $\mathrm{p}=0.718)$, shoulder $(p=0.472)$, and leg $(p=0.378)$, did not change significantly when oils were supplemented.

Chemical composition of muscles was not affected by oil supplementation (Table 4); however, a tendency towards increased CP in MS ( $p=0.097$ ) was observed when lambs were fed rapeseed oil in comparison with linseed oil. The weights 
Table 3. Animal performance, carcass characteristic and carcass components of major cuts of lambs

\begin{tabular}{|c|c|c|c|c|c|}
\hline Item & Control & Rapeseed oil & Linseed oil & SEM & $p$-value \\
\hline Daily feed intake (kg/d) & 1.15 & 1.2 & 1.2 & - & - \\
\hline Initial live weight (kg) & 19.9 & 19.7 & 19.5 & 0.46 & 0.941 \\
\hline Slaughter weight $(\mathrm{kg})$ & 35.7 & 35.7 & 35.7 & 0.12 & 0.955 \\
\hline Days of fattening & 56.3 & 54.3 & 55.5 & - & - \\
\hline $\operatorname{ADG}(g / d)$ & 280 & 295 & 293 & 8.7 & 0.816 \\
\hline FCR & 4.1 & 4.1 & 4.1 & 0.13 & 0.982 \\
\hline Hot carcass weight $(\mathrm{kg})$ & 14.3 & 15.1 & 14.7 & 0.16 & 0.100 \\
\hline Right half of carcass $(\mathrm{kg})$ & 6.4 & 7.1 & 6.9 & 0.12 & 0.060 \\
\hline $\mathrm{DP}(\%)$ & $40.0^{a}$ & $42.4^{b}$ & $41.2^{\mathrm{ab}}$ & 0.40 & 0.039 \\
\hline Loin $(\mathrm{kg})$ & 0.3 & 0.4 & 0.3 & 0.01 & 0.718 \\
\hline Shoulder (kg) & 1.2 & 1.1 & 1.1 & 0.02 & 0.472 \\
\hline $\operatorname{Leg}(\mathrm{kg})$ & 1.7 & 1.8 & 1.7 & 0.05 & 0.378 \\
\hline
\end{tabular}

SEM, standard error of mean; ADG, average daily gain; FCR, feed conversion ratio; DP, dressing percentage (hot carcass weight $\times 100 /$ slaughter weight).

${ }^{a b}$ Means in a row with different superscripts are significantly different $(p<0.05)$.

of internal organs (liver [ $p=0.674]$, heart [ $p=0.726]$, and kidneys [ $p>0.100]$ ) did not differ between the three treatments.

\section{Oil supplementation effects on saturated fatty acids}

The concentrations of C15:0 ( $p=0.003), C 16: 0(p=0.045)$, $\mathrm{C} 17: 0(\mathrm{p}=0.027), \mathrm{C} 21: 0(\mathrm{p}<0.001), \mathrm{C} 24: 0(\mathrm{p}<0.001)$, and as a consequence, of total SFA ( $p=0.049$ ) were significantly lower in MLD in the group receiving linseed oil than in the control group (Table 5). When linseed oil was added to the diet, C24:0 concentration in MS was significantly lower $(\mathrm{p}=0.006)$ as compared to the control ration (Table 6). Total SFA in MS was not influenced by oil supplementations $(\mathrm{p}=0.975)$.

\section{Oil supplementation effects on monounsaturated fatty} acids

Linseed oil supplementation significantly decreased the con-

Table 4. Chemical composition of muscles ( $\mathrm{g} / 100 \mathrm{~g}$ ) and internal organs weight $(\mathrm{g})$

\begin{tabular}{lccccc}
\hline Item & Control & Rapeseed oil & Linseed oil & SEM & p-value \\
\hline MLD & & & & & \\
$\quad$ Dry matter & 23.9 & 23.9 & 23.9 & 0.14 & 0.994 \\
Crude protein & 19.3 & 19.1 & 19.1 & 0.14 & 0.818 \\
Crude ash & 1.1 & 1.1 & 1.1 & 0.01 & 0.317 \\
$\quad$ Crude fat & 2.4 & 2.4 & 2.7 & 0.10 & 0.468 \\
MS & & & & & \\
Dry matter & 24.0 & 24.5 & 23.1 & 0.17 & 0.471 \\
Crude protein & 19.4 & 19.7 & 18.9 & 0.14 & 0.097 \\
Crude ash & 1.1 & 1.1 & 1.1 & 0.01 & 0.121 \\
Crude fat & 1.9 & 2.6 & 2.4 & 0.16 & 0.286 \\
Internal organs & & & & & \\
Liver & 618 & 611 & 642 & 14.1 & 0.674 \\
Heart & 160 & 165 & 168 & 4.0 & 0.726 \\
Right kidney & 46.0 & 50.0 & 46.2 & 2.04 & 0.623 \\
Left kidney & 47.3 & 47.3 & 46.5 & 1.57 & 0.972 \\
\hline
\end{tabular}

SEM, standard error of mean; MLD, longissimus dorsi muscle; MS, semitendinosus muscle. centration of $\mathrm{C} 16: 1(\mathrm{p}=0.024), \mathrm{C} 17: 1(\mathrm{p}<0.001), \mathrm{C} 18: 1$ cis-9 $(\mathrm{p}=0.006)$ and total MUFA ( $\mathrm{p}=0.011)$ in MLD compared to rapeseed oil and control groups (Table 5). The addition of rapeseed oil to the diets significantly decreased C15:1 concentration $(\mathrm{p}=0.006)$ in MLD in comparison to control and linseed oil rations. Moreover, the addition of linseed oil to lamb diets significantly increased C24:1 contents $(p=0.003)$ in MS than in control and did not influence other MUFA $(\mathrm{p}>0.100)$ (Table 6).

\section{Oil supplementation effects on polyunsaturated fatty acids}

The concentrations of ALA $(\mathrm{p}<0.001)$, EPA $(\mathrm{p}=0.010)$ and total $n$-3 PUFA ( $\mathrm{p}=0.011$ ) in MLD were significantly higher when linseed oil was provided in comparison to control and lambs fed rapeseed oil (Table 5). In turn, the content of C20:3 in MLD was significantly lower $(p=0.001)$ in animals fed linseed oil than in control ones. Oil supplementations to animal diets caused significantly increased concentration of DHA $(p=0.018)$ in MLD when compared to the control diet. The addition of linseed oil to the diets significantly increased concentrations of ALA ( $p<0.001)$, EPA ( $p=0.007)$ as well as total $n-3$ PUFA ( $\mathrm{p}=0.001)$ and sum of PUFA $(\mathrm{p}=0.004)$ in MS compared to control and rapeseed oil groups (Table 6). Further, the addition of oils to the rations significantly increased DHA concentration in MS $(p=0.008)$.

\section{Oil supplementation effects on $\mathrm{n}: 6 / \mathrm{n}: 3$ and PUFA/SFA} ratios and $\Delta^{9}$-desaturase index

A tendency towards a lower $\mathrm{n}: 6 / \mathrm{n}: 3$ ratio $(\mathrm{p}=0.054)$ in MLD was observed when lambs were fed linseed oil in comparison to control and rapeseed oil diets (Table 5). The addition of linseed oil to the diets significantly increased PUFA/SFA ratio $(\mathrm{p}=0.002)$ in MLD as compared to control and rapeseed oil groups (Table 5). The $\Delta^{9}$-desaturase index was the lowest in 
$\operatorname{MLD}(\mathrm{p}=0.062)$ and MS $(\mathrm{p}=0.013)$ when lambs were fed linseed oil (Tables 5, 6).

\section{DISCUSSION}

Animal performance

Generally, the type of dietary fat supplementation has no effect on growth performance and carcass traits of lambs or goats $[21,22,10]$. However, in the present study, rapeseed oil supplementation was associated with a higher DP, carcass and half carcass weights. These results can be explained by the high energy level, crude fat concentration in lamb diets and higher DM intake. These observations are consistent with the results of Peng et al [9], who reported beneficial effects of rapeseed oil supplementation to diet on DP and carcass weight.

No differences were identified between control and treatment groups in terms of muscle (MLD, MS) chemical composition. Similar results were reported in relation to the effect of supplementation of different forms of sunflower products on DM, $\mathrm{CP}$, fat and ash contents in muscles [10].

Table 5. The fatty acid composition of longissimus dorsi muscle in lambs (mg FAME/100 g muscle)

\begin{tabular}{|c|c|c|c|c|c|}
\hline Fatty acid & Control & Rapeseed oil & Linseed oil & SEM & $p$-value \\
\hline \multicolumn{6}{|l|}{ SFA } \\
\hline $\mathrm{C} 10: 0$ & nd & nd & nd & - & - \\
\hline C14:0 & 50.1 & 43.0 & 33.7 & 3.69 & 0.196 \\
\hline C15:0 & $21.9^{\mathrm{a}}$ & $19.7^{\mathrm{ab}}$ & $17.0^{\mathrm{b}}$ & 0.68 & 0.003 \\
\hline C 16:0 & $514^{\mathrm{a}}$ & $449^{a b}$ & $388^{b}$ & 21.6 & 0.045 \\
\hline C17:0 & $63.2^{\mathrm{a}}$ & $48.6^{\mathrm{ab}}$ & $45.8^{b}$ & 3.04 & 0.027 \\
\hline C18:0 & 473 & 411 & 382 & 20.5 & 0.188 \\
\hline$C 20: 0$ & 6.6 & 5.3 & 6.4 & 0.50 & 0.565 \\
\hline $\mathrm{C} 21: 0$ & $9.0^{\mathrm{a}}$ & $7.4^{\mathrm{a}}$ & $4.5^{b}$ & 0.55 & $<0.001$ \\
\hline$C 24: 0$ & $4.2^{\mathrm{a}}$ & $3.0^{a b}$ & $2.6^{b}$ & 0.20 & $<0.001$ \\
\hline$\Sigma S F A^{1)}$ & $1,142^{\mathrm{a}}$ & $987^{\mathrm{ab}}$ & $880^{b}$ & 45.6 & 0.049 \\
\hline \multicolumn{6}{|l|}{ MUFA } \\
\hline C14:1 cis-9 & 8.9 & 7.3 & 5.9 & 0.56 & 0.078 \\
\hline C15:1 cis 10 & $3.7^{\mathrm{a}}$ & $1.9^{b}$ & $2.1^{\mathrm{a}}$ & 0.28 & 0.006 \\
\hline C16:1 cis-9 & $27.4^{\mathrm{a}}$ & $25.7^{\mathrm{a}}$ & $18.0^{b}$ & 1.62 & 0.024 \\
\hline C17:1 cis- 10 & $13.3^{\mathrm{a}}$ & $9.8^{\mathrm{b}}$ & $7.0 \mathrm{c}$ & 0.73 & $<0.001$ \\
\hline C18:1 trans- 11 (VA) & 23.4 & 20.5 & 30.6 & 2.55 & 0.251 \\
\hline C18:1 cis-9 & $792 a$ & $766^{\mathrm{a}}$ & $582^{b}$ & 33.00 & 0.006 \\
\hline C24:1 cis-15 & 8.3 & 8.8 & 10.1 & 0.32 & 0.053 \\
\hline$\Sigma$ MUFA $^{2)}$ & $877^{\mathrm{a}}$ & $840^{\mathrm{a}}$ & $656^{\mathrm{b}}$ & 35.4 & 0.011 \\
\hline \multicolumn{6}{|l|}{ PUFA } \\
\hline C18:2 n- 6 trans -9 trans -12 & 6.4 & 6.1 & 11.5 & 1.02 & 0.118 \\
\hline C18:2 n-6 cis-9 cis-12 (LA) & 110 & 101 & 108 & 2.9 & 0.449 \\
\hline C18:2 cis-9 trans-11 (CLA) & 8.6 & 7.0 & 8.4 & 0.66 & 0.565 \\
\hline C18:3n-3 (ALA) & $10.2^{\mathrm{a}}$ & $11.1^{\mathrm{a}}$ & $28.4^{b}$ & 2.33 & $<0.001$ \\
\hline$C 20: 3(n-6)$ & $3.4^{\mathrm{a}}$ & $2.9^{b}$ & $2.3^{c}$ & 0.12 & $<0.001$ \\
\hline$C 20: 3(n-3)$ & $44.4^{\mathrm{a}}$ & $38.0^{\mathrm{ab}}$ & $32.0^{b}$ & 1.64 & 0.001 \\
\hline$C 20: 5$ (n-3 EPA) & $3.4^{\mathrm{a}}$ & $4.6^{\mathrm{ab}}$ & $6.4^{b}$ & 0.46 & 0.010 \\
\hline $\mathrm{C} 22: 6(n-3 \mathrm{DHA})$ & $2.2^{\mathrm{a}}$ & $3.3^{b}$ & $3.4^{b}$ & 0.21 & 0.018 \\
\hline$\Sigma$ PUFA $^{3)}$ & 180 & 167 & 192 & 4.6 & 0.093 \\
\hline$\Sigma$ PUFA $n-6^{4)}$ & 120 & 110 & 122 & 3.1 & 0.269 \\
\hline$\Sigma$ PUFA $n-3^{5)}$ & $60.2^{\mathrm{a}}$ & $57.0^{\mathrm{a}}$ & $70.2^{b}$ & 1.98 & 0.011 \\
\hline$n-6 / n-3$ & 2.0 & 1.9 & 1.7 & 0.06 & 0.054 \\
\hline PUFA/SFA & $0.16^{\mathrm{a}}$ & $0.17^{\mathrm{a}}$ & $0.21^{b}$ & 0.009 & 0.002 \\
\hline$\Delta^{9}$-desaturase index & 0.44 & 0.47 & 0.42 & 0.007 & 0.062 \\
\hline
\end{tabular}

FAME, fatty acid methyl esters; SEM, standard error of mean; SFA, saturated fatty acids; nd, not detectable; MUFA, monounsaturated fatty acids; VA, vaccenic acid; PUFA, polyunsaturated fatty acids; LA, linoleic acid; CLA, conjugated linoleic acid; ALA, linolenic acid; EPA, eicosapentaenoic acid; DHA, docosahexaenoic acid;

1) Sum of SFA: C14:0; C15:0; C16:0; C17:0; C18:0; C20:0; C21:0; C24:0.

2) Sum of MUFA: C14:1 cis-9; C15:1 cis-10; C16:1 cis-9; C17:1 cis-10; C18:1 (VA); C18:1 cis-9; C24:1 cis-15.

3) Sum of PUFA: C18:2 trans-9 trans-12; C18:2 (LA); C18:3 (ALA); C20:3 n-6; C20:3 n-3; C20:5 (EPA); C22:6 (DHA).

4) Sum of PUFA $n-6$ : C18:2 trans-9 trans-12; C18:2 (LA); C20:3 n-6.

${ }^{5)}$ Sum of PUFA $n-3:$ C 18:3 (ALA); C20:3 n-3; C20:5 (EPA); C22:6 (DHA).

abc Means in a row with different superscripts are significantly different $(p<0.05)$. 
Table 6. The fatty acid composition of semitendinosus muscle in lambs (mg FAME/100 g muscle)

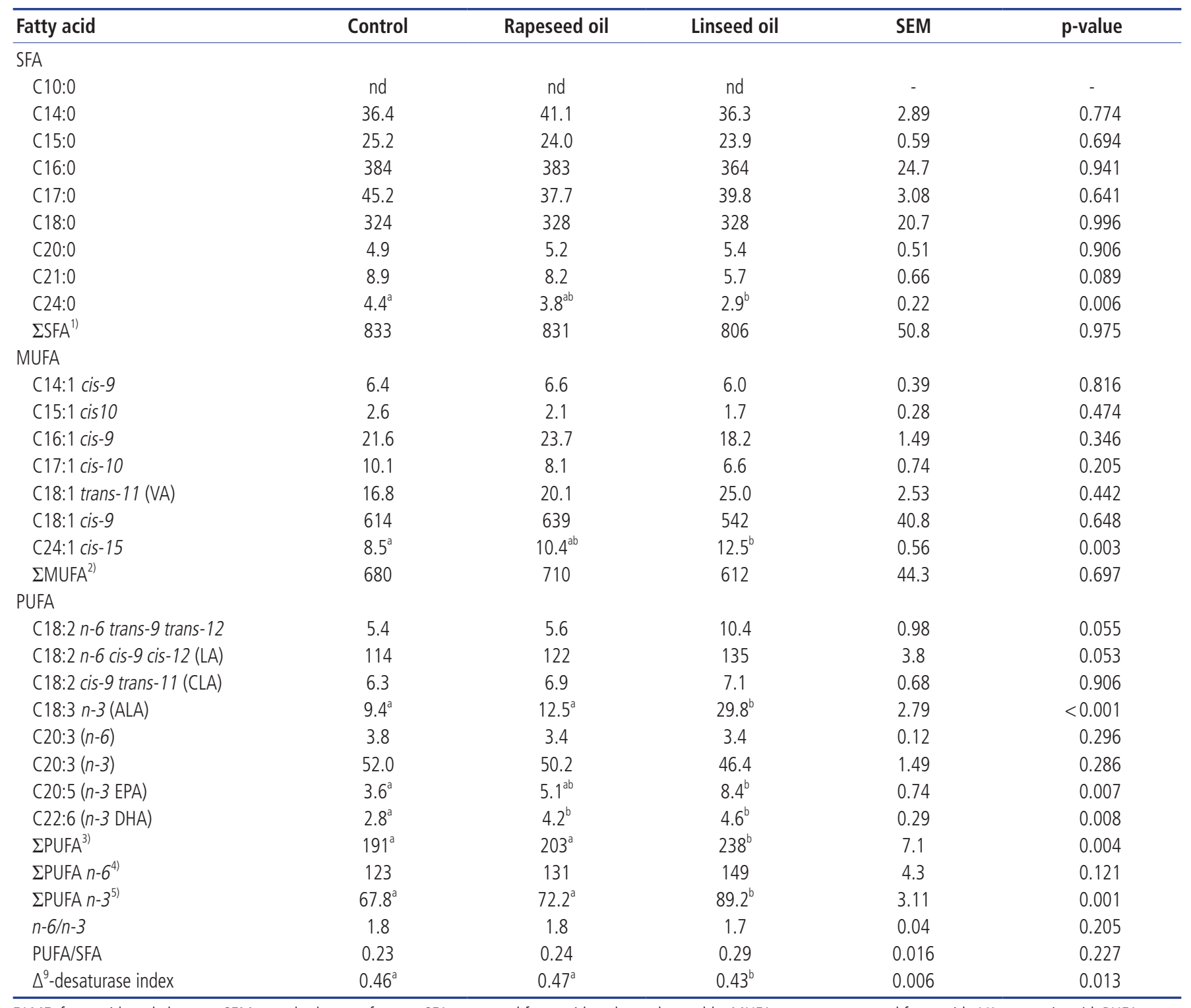

FAME, fatty acid methyl esters; SEM, standard error of mean; SFA, saturated fatty acids; nd, not detectable; MUFA, monounsaturated fatty acids; VA, vaccenic acid; PUFA, polyunsaturated fatty acids; LA, linoleic acid; CLA, conjugated linoleic acid; ALA, linolenic acid; EPA, eicosapentaenoic acid; DHA, docosahexaenoic acid.

1) Sum of SFA: C14:0; C15:0; C16:0; C17:0; C18:0; C20:0; C21:0; C24:0.

2) Sum of MUFA: C14:1 cis-9; C15:1 cis-10; C16:1 cis-9; C17:1 cis-10; C18:1 (VA); C18:1 cis-9; C24:1 cis-15.

3) Sum of PUFA: C18:2 trans-9 trans-12; C18:2 (LA); C18:3 (ALA); C20:3 n-6; C20:3 n-3; C20:5 (EPA); C22:6 (DHA).

4) Sum of PUFA $n-6$ : C18:2 trans-9 trans-12; C18:2 (LA); C20:3 n-6.

5) Sum of PUFA $n-3:$ C 18:3 (ALA); C20:3 n-3; C20:5 (EPA); C22:6 (DHA).

${ }^{\text {ab }}$ Means in a row with different superscripts are significantly different $(p<0.05)$.

\section{Meat fatty acid composition}

The content and profile of specific FA in meat are important factors in assessing its nutritional quality. The FA profiles of adipose tissue and muscles are determined by de novo lipogenesis, desaturation as well as ration lipid composition, feeding duration and difference in the utilization of various FA by the animal body $[8,11]$.

Saturated fatty acids: Linseed oil supplementation reduced SFA (C15:0, C16:0, C17:0, C21:0, C24:0) in MLD, but decreased only C24:0 concentration in MS. Linseed oil supplementation lowers SFA levels, especially C15:0, C16:0, and C17:0, which is desirable, because this FA is the major hypercholesterolemic FA and it is associated with a higher risk of cardiovascular diseases and type 2 diabetes [2]. According to Yang et al [23], decreased SFA in muscles of ruminants fed linseed oil in the ration was presumably caused by de novo synthesis inhibition by a higher percentage of exogenous FA in the metabolic pool. Additionally, SFA reduction in muscles derived from lambs 
fed linseed oil may also reflect an incomplete biohydrogenation process, as an effect of the high consumption of unprotected fats rich in linolenic acid. Czauderna et al [24] reported a reduction in the concentration of SFA in lamb longissimus dorsi and biceps femoris muscles after supplementing control diet with linseed oil. These authors suggested that the oil stimulated peroxidation damage and/or catabolism of FA. Our results are consistent with those of Gallardo et al [22], who observed a greater decrease in C15:0, C16:0, C17:0, and C24:0 concentrations in intramuscular fat of lambs sucking ewes and receiving diets supplemented with linseed oil than in the control animals. On the other hand, Ebrahimi et al [13] did not find significant changes in SFA concentration in the subcutaneous adipose tissue of goats fed diet with different levels of linseed oil.

Monounsaturated fatty acids: MUFA contain a single double bond in the cis- or trans-configuration. Linseed oil inclusion into diet causes changes in the MUFA profile in MLD, in particular reduces oleic acid (C18:1 cis-9), which is the main MUFA. Bessa et al [12] also found that linseed oil in diets decreased C18:1 cis-9 concentration in longissimus thoracis in comparison to un-supplemented diets. Oleic acid reduction in ruminant tissues is often associated with PUFA supplementation in the ration [25]. Such situation can be due to either the absence of C18:0 (stearic acid) desaturated by $\Delta^{9}$-desaturase or the inhibition of enzyme activity by high PUFA contents [26]. In our experiment, the quantity of stearic acid in the ration was low, thus its origin here was primarily derived from endogenous de novo synthesis, and as an end-product of $\mathrm{C} 18$ PUFA biohydrogenation in the rumen [12]. In PUFA-supplemented ruminant diets, de novo FA synthesis is reduced; PUFA biohydrogenation becomes incomplete, and biohydrogenation intermediates start to accumulate, causing lower $\mathrm{C} 18: 0$ yield, as shown in the current study. Moreover, Mosley et al [27] documented in the in vitro study a direct octadeceonoate cis-trans isomerization in mixed rumen bacteria, capable of converting oleic acid into trans-11 as well as other trans isomers. In our study, insignificant increase of C18:1 trans 11 in MLD and MS was observed when lambs were fed linseed oil in comparison to other diet. However, C18:0 reduction in MLD of lambs fed linseed oil was small. In contrast to our findings, Gallardo et al [22] did not find any significant changes in C18:1 cis-9 concentrations of intramuscular fat of lamb sucking ewes fed diets supplemented with linseed oil.

Polyunsaturated fatty acids: Lipids in vegetable oils are characterized by a high content of USFAs. Feeding vegetable oils, such as linseed, rapeseed or fish oils decreases SFA concentration in meat and increases the content of valuable PUFA especially ALA, EPA, DHA. Feeding strategies that induce a decrease in saturated lipids and an increase in $n-3$ FA of intramuscular fat would improve the value of lamb meat. Conversion capacity of C18:3n-3 to health-promoting long chain $n-3$ PUFA is limited in humans [2], which reinforce the significance of its dietary supply. The increase in the concentration of long chain n-3 PUFA coincided with increase in dietary C18:3 n-3 concentration of linseed oil rations. This pattern was consistent with other trials where lambs were fed oils rich in C18:3 $n-3[28,13]$. Lambs fed diet containing linseed oil accumulated a significantly higher amount of total $n-3$ PUFA in their muscles than lambs fed other diets. Long chain $n$-3 PUFA in muscles are metabolic products of $\mathrm{C} 18: 3 n-3$. Competition between C18:3n-3 and C18:2 n-6 for desaturation and elongation enzymes may affect the conversion to long chain derivatives, while the high concentration of $n$ - 3 PUFA is likely due to the preference of these enzymes for C18:3n-3. On the other hand, Bessa et al [12] and Gallardo et al [22] showed that diet supplementation with linseed oil, high in C18:3 $n-3$, was coupled with decreased amounts of long chain $n$-3 PUFA in longissimus thoracis and intramuscular fat, suggesting an inhibition of C18:3 n-3 metabolism. Bessa et al [12] explained that this inhibition could have been caused by the repression of gene expression mediated by linolenic acid.

In our experiment, there was a decrease in the amounts of C20:3 n-6 in MLD muscle in lambs fed linseed oil. According to Nuernberg et al [29], this could be explained by the lower total FA concentration in the grass-based diet, allowing long chain phospholipid FA to dominate total lipids. Hence, it cannot be precluded that in our study the amount of total FA in meadow hay varied, all the more that lamb fattening lasted more than 50 days.

Nutritional quality of meat: The $n-6 / n-3$ and PUFA/SFA ratios are used to evaluate the nutritional value of fat for human consumption. The $\mathrm{n}: 6 / \mathrm{n}: 3$ ratio is strongly dependent on the FA profile of the ration fed to ruminants. This ratio is particularly beneficial in the meat of ruminants that consume forages or oilseed with an increased C18:3 content [2]. Lowering the n-6/n:3 coefficient in food production has been recommended to prevent or modulate certain human diseases, and it should range between 1 and 4 [4]. Our results were within this range, but the n:6/n:3 ratio found in MLD and MS was the lowest when lambs were fed linseed oil. These results are consistent with findings of Jerónimo et al [28], who reported that the n:6/n:3 ratio in MLD of lambs was significantly decreased when dietary sunflower oil was replaced with linseed oil. In the group with linseed oil, $59.55 \%$ of total $n$-3 PUFA were long chain $n$-3 PUFA (C20:3, C20:5, and C22:6). Ebrahimi et al [13] believed that this could be important considering that health benefits of $n$-3 PUFA are predominantly associated with long chain $n$-3 PUFA, as C18:3n-3 metabolism is restricted in humans.

Usually lamb meat has a high SFA content and low PUFA/ SFA values. Increasing the PUFA concentration in the ration, by including a source rich in either $n-6$ or $n$ - 3 PUFA generally improves the PUFA/SFA ratio. This was also observed in the present study, and in oil diets, the PUFA/SFA ratio was 
lower than 0.45 , so as recommended by the $\mathrm{WHO} / \mathrm{FAO}$ [4].

\section{CONCLUSION}

According to the results of the present study, FA profile in muscles was depended on oils supplemented to lamb diets. Additionally, the supplementation of linseed oil to diets seems to reduce the concentration of SFA and increase the concentration of $n$ - 3 PUFA, particularly ALA, EPA, and DHA in meat. The $n-6 / n-3$ ratio is an important nutritional factor, and its value has been favorably decreased below 2 , thereby achieving an important target related to human health. Due to these changes carcass FA profile was improved, and so enhanced lamb meat healthy properties.

\section{CONFLICT OF INTEREST}

We certify that there is no conflict of interest with any financial organization regarding the material discussed in the manuscript.

\section{ACKNOWLEDGMENTS}

The study was supported by the funds granted by the Polish Ministry of Science and Higher Education under the Project No. N N311 110138 entitled 'The effect of tannins and oils addition to sheep diets on microorganism population, rumen fermentation, enzymatic activity of pancreatic-biliary juice and lamb quality'.

\section{REFERENCES}

1. Givens DI. The role of animal nutrition in improving the nutritive value of animal-derived foods in relation to chronic disease. Proc Nutr Soc 2005;64:395-402.

2. Chikwanha OC, Vahmani P, Muchenje V, Dugan MR, Mapiye C. Nutritional enhancement of sheep meat fatty acid profile for human health and wellbeing. Food Res Int 2018;104:2538

3. Sales-Campos H, de Souza PR, Peghini BC, da Silva JS, Cardoso $\mathrm{CR}$. An overview of the modulatory effect of oleic acid in health and disease. Mini Rev Med Chem 2013;13:201-10.

4. WHO/FAO (World Health Organization/Food and Agriculture Organization). Diet, nutrition and the prevention of chronic diseases. Geneva, Switzerland: WHO Technical Report Series; 2003.

5. Givens DI, Kliem KE, Gibbs RA. The role of meat as a source of $\mathrm{n}-3$ polyunsaturated fatty acids in the human diet. Meat Sci 2006;74:209-18.

6. Wahle, KWJ, Heys SD, Rotondo D. Conjugated linoleic acids: are they beneficial or detrimental to health? Prog Lipid Res 2004;43:553-87.
7. Corl BA, Baumgard LH, Dwyer DA, Griinari JM, Phillips BS, Bauma DE. The role of $\Delta 9$ desaturase in the production of cis- 9 , trans-11 CLA. J Nutr Biochem 2001;12:622-30.

8. Oliveira DM, Ladeira MM, Chizzotti ML, et al. Fatty acid profile and qualitative characteristic of meat from zebu steers fed with different oilseeds. J Anim Sci 2011;89:2546-55.

9. Peng YS, Brown MA, Wu JP, Liu Z. Different oilseed supplements alter fatty acid composition of different adipose tissues of adult ewes. Meat Sci 2010;85:542-9.

10. Majewska MP, Pająk JJ, Skomiał J, Kowalik B. The effect of different forms of sunflower products in diets for lambs and storage time on meat quality. Anim Feed Sci Technol 2016;222:22735.

11.Realini CE, Bianchi G, Bentancur O, Garibatto G. Effect of supplementation with linseed or a blend of aromatic spices and time on feed on fatty acid composition, meat quality and consumer liking of meat from lambs fed dehydrated alfalfa or corn. Meat Sci 2017;127:21-9.

12. Bessa RJB, Alves SP, Jerónimo E, Alfaia CM, Prates JM, SantosSilva J. Effect of lipid supplements on ruminal biohydrogenation intermediates and muscle fatty acids in lambs. Eur J Lipid Sci Technol 2007;109:868-78.

13.Ebrahimi M, Rajion MA, Goh YM, Sazili AQ, Schonewille J. Effect of linseed oil dietary supplementation on fatty acid composition and gene expression in adipose tissue of growing goats. Biomed Res Int 2013;2013:194625

14.IZ-PIB INRA. Feeding recommendation for ruminants and feed tables. Krakow Poland: National Research Institute of Animal Production in Balice, Poland; 2014.

15. FAO (Food and Agricultural Organization). Animal production and health paper. Guidelines for slaughtering, meat cutting and further processing. Rome, Italy: Agriculture and Consumer Protection; 1991.

16. AOAC (Association of Official Analytical Chemists). Official Methods of Analysis, 15th edn. Arlington, VA, USA: AOAC International; 2011.

17.van Soest PJ, Robertson JB, Lewis BA. Methods for dietary fiber, neutral detergent fiber, and nonstarch polysaccharides in relation to animal nutrition. J Dairy Sci 1991;74:3583-97.

18. Folch J, Less M, Stanely GHS. A simple method for the isolation and purification of total lipides from animal tissue. J Biol Chem 1957;226:497-509.

19. Czauderna M, Kowalczyk J, Krajewska KA, Rozbicka AJ, Michalski JP. Dietary selenite and conjugated linoleic acid isomers influence fatty acid concentrations in the liver and femoral muscles of rats. J Anim Feed Sci 2009;18:564-81.

20. Brogna DMR, Nasri S, Ben Salem H, et al. Effect of dietary saponins from Quillaja saponaria $\mathrm{L}$. on fatty acid composition and cholesterol content in muscle Longissimus dorsi of lambs. Animal 2011;5:1124-30.

21. Karami M, Ponnampalam EN, Hopkins DL. The effect of palm oil or canola oil on feedlot performance, plasma and tissue 
fatty acid profile and meat quality in goats. Meat Sci 2013;94: 165-9.

22. Gallardo B, Manca MG, Mantecón AR, Nudda A, Manso T. Effects of linseed oil and natural or synthetic vitamin E supplementation in lactating ewes' diets on meat fatty acid profile and lipid oxidation from their milk fed lambs. Meat Sci 2015; 102:79-89.

23. Yang YT, Baldwin RL, Garrett WN. Effects of dietary lipid supplementation on adipose tissue metabolism in lambs and steers. J Anim Sci 1978;47:686-90.

24. Czauderna M, Kowalczyk J, Niedźwiedzka KM, Wąsowska I, Pająk JJ. The effect of selenium and linseed oil growth of sheep and content of selected fatty acids in M. longissimus dorsi. J Anim Feed Sci 2004;13(Suppl 1):303-6.

25. Bessa RJB, Portugal PV, Mendes IA, Santos-Silva J. Effect of lipid supplementation on growth performance, carcass and meat quality and fatty acid composition of intramuscular lipids of lambs fed dehydrated lucerne or concentrate. Livest Prod Sci 2005;96:185-94.

26. Sampath H, Ntambi JM. Polyunsaturated fatty acid regulation of genes of lipid metabolism. Annu Rev Nutr 2005;25;317-40.

27. Mosley EE, Powell GL, Riley MB, Jenkins TC. Microbial biohydrogenation of oleic acid to trans isomers in vitro. J Lipid Res 2002;43:290-6.

28. Jerónimo E, Alves SP, Prates JAM, Santos-Silva J, Bessa RJB. Effect of dietary replacement of sunflower oil with linseed oil on intramuscular fatty acids of lamb meat. Meat Sci 2009;83: 499-505.

29. Nuernberg K, Dannenberger D, Nuernberg G, et al. Effect of a grass-based and a concentrate feeding system on meat quality characteristics and fatty acid composition of longissimus muscle in different cattle breeds. Livest Prod Sci 2005;94:137-47. 